L. E. M. de Boer

Institute of Genetics, University of Uirecht, Utrecht, The Netherlands

Received 10 March 1971

\section{Marked Chromosomes Associations in Catarrhine Monkeys, with a Note on Chromosome Associations in Other Primate Groups}

In metaphase figures, obtained from cultures of whole blood, associations of the marked chromosomes were found in species of the following genera: Macaca, Cercocebus, Cercopithecus and Symphalangus. The different types of these associations are discussed. A note is given on chromosome: associations found in some species of Prosimii and Platyrrhinae.

In numerous studies on the cytogenetics of the Old World monkeys that appeared during the past 15 years, the term "marked chromosomes" has become a concept, indicating a pair of chromosomes, presenting a deep secondary constriction, connected usually with large satellites in one of the chromosome-arms. In all species of the families Cercopithecidae and Hylobatidae these elements are present in every metaphase figure. Since only a single pair occurs per cell, the homologues can be easily recognized.

In the Cercopithecidae three morphologically distinct types of marked chromosomes can be distinguished:

Submetacentric (arm ratio $\pm 1 \cdot 3$ ), with the constriction in the short arm [cf. Figure 1(b)] (All species of the genera Macaca, Cercocebus and Papio; subfamily Papinae, Chiarelli, 1966a.)

Subterminal, with the constriction in the long arm [Figure 1(a)] (In the numerous species and subspecies of Cercopithecus subfamily Cercopithecinae, including the genus Erythrocebus; Chiarelli, 1963.)

The constant morphology of the marked chromosomes in this group, in which the diploid chromosome number varies from 54 to 72 , is striking.

Almost metacentric [Figure 1(c)] (subfamily Colobinae, as far as karyotypes are known: several species of Colobus, Presbytis and Nasalis larvatus Wurmbs, 1821).

According to Chiarelli (1966b), who measured a number of marked chromosomes of various Old World monkeys, the marked arm in the three types is of about the same size, whereas the not-marked arm varies in length between the different groups.

In the gibbons (family Hylobatidae) two types can be distinguished:

All investigated species of Hylobates have matecentric marked chromosomes, of approximately the same length as those in the Colobinae (Chiarelli, 1 966b) [Figure $1(\mathrm{c})]$.

The second type, that of Symphalangus syndactylus, is not always regarded as a marked chromosome comparable to that secn in the other catarrhine monkeys (Chiarelli, 1966b). As Figure l (c) shows, they are acrocentric, bearing small satellites on their short arms. This is the only acrocentric pair in the karyotype of this small animal $(2 n=50)$, and there are no other chromosomes with secondary constrictions. For these reasons they can be considered as marked chromosomes. The only difference is that the satellites are much smaller. (In our material of Symphalangus syndactylus there is some doubt about the length of the short arms; it varies markedly depending on the grade of spiralization of the achromatic region.)

In the literature there are two notes on the existence of associations between marked chromosomes of catarrhine monkeys. The first observation was made by Klinger (1963) in metaphase plates of Symphalangus syndactylus (". . . both partners bear satellites on the 
Figure 1. Schematic representation of marked chromosome types in the families Cercopithecidae ( $a$, $b, c)$ and Hylobatidae (d, e), (a) Cercopithecus species; (b) Genera Macaca, Cercocebus and Papio; (c) Subfamily Colobinae; (d) Genus Hylobatus; (e) Symphalangus syndactylus.

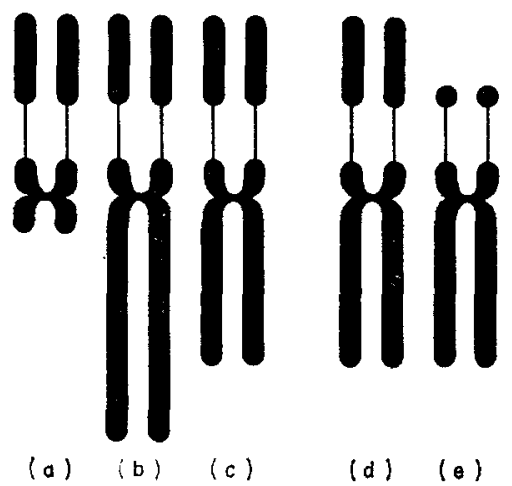

short arms and some metaphase figures showed satellite association".), whereas Fernandez-Donoso (1970) recorded the phenomenon in Macaca fascicularis.

In metaphase figures, obtained from cultures of whole blood (de Boer, 1971) of a number of Old World monkeys, associations of the marked chromosomes were often observed in the following species: Macaca fascicularis (Raffles, 1821), M. nemestrina (Raffles, 1821), Cercocebus torquatus (Kerr, 1792), Cercopithecus talapoin (Schreeber, 1774), C. mitis albogularis (Sykes, 1831), C. hamlyni (Pocock, 1907) and Symphalangus syndactylus (Raffles, 1821). The highest associative frequency was found in Symphalangus syndactylus; more than $40 \%$ of 95 metaphase plates showed a characteristic configuration of the two chromosomes in question (Plate 1 -parts $7,8,9$ ). When these elements lie independently in the metaphase plate they bear small, but clearly visible satellites. If they are associated however, the satellites are no longer recognizable and the chromosomes seem to be connected by thread-like structures.

Some association types in Cercocebus torquatus are shown in Plate 1 (parts 1, 2, 3). It seems clear that the associations are formed by the achromatic regions rather than by the satellites; though less frequently, the same types were found also in Macaca fascicularis and $M$. nemestrina.

Association between the acrocentric type of marked chromosomes are shown in Plate 1 (parts 4, 5,6) (Cercopithecus talapoin). Again it is clear that they are concentrated on the achromatic regions. A similar situation was found in $C$. mitis albogularis and $C$. hamlyni.

In all species considered only the marked chromosomes were involved in associations. Even in the three Cercopithecus species, characterized by the presence of many acrocentrics (none of which satellitcd), no other associations were found. In this way, contrary to the situation in man, where associations between not-homologous chromosomes also exist, in our material, we are always dealing with associations between homologous chromosome parts. In the anthropoid apes, where no marked chromosomes occur, several satellitebearing acrocentric chromosomes are found, forming associations of the human type (Chimpanzee six pairs, Gorilla eight pairs, Orang-utan ten pairs) (Hannerton, Klinger, Mutton \& Lang, 1963).

The observation that the associations are concentrated on the achromatic regions, and not on the satellites, is in agreement with the experiences of Van Hemel (1971) for human associations. Whether the thread-like structures in Symphalangus syndactylus associations are comparable to those sometimes found in man (Zang \& Back, 1968) is not certain.

The physiological function of the achromatic region in the marked chromosomes might lie in the organization of the nucleolus during interphase, but this has never been proved. Nevertheless, the occurrence of asssociations between these regions fortifies this idea, the 
Plate 1. Details of metaphase figures (obtained from blood cultures) showing associations between three different types of marked chromosomes of catarrhine monkeys (4000X): 1-3: Cercocebus torquatus (Kerr, 1792); 4-6: Cercopithecus: talapoin (Schreeber, 1774); 7-9: Symphalangus syndactylus (Raffles, 1321 .

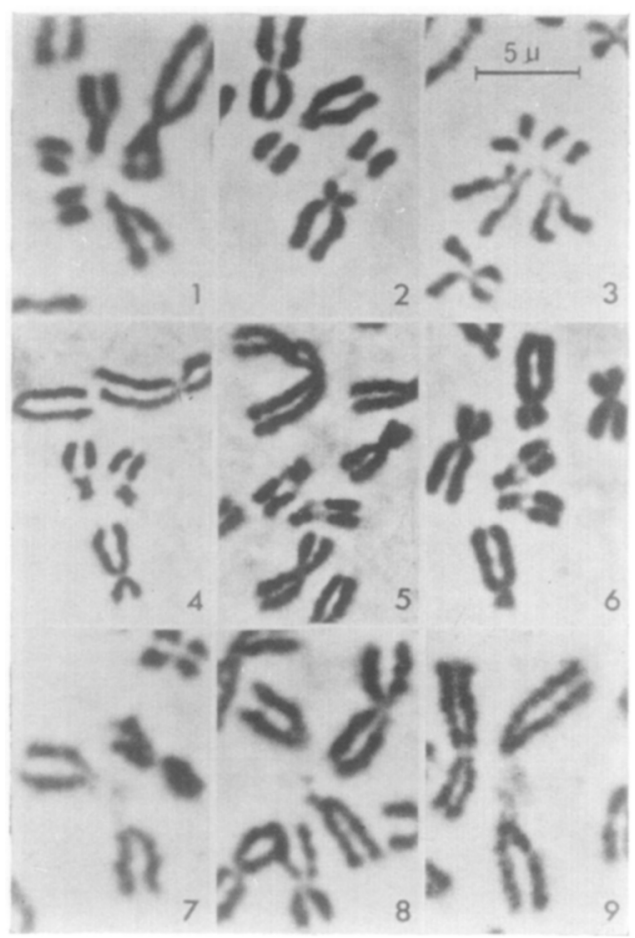


more so since one comes across the same situation in the human material (Ohno, 1961). At any rate, the evidence on the associations in species with different types of marked chromosomes suggests, in addition to the morphological homologization (Chiarelli, $1966 b$; Egozcue \& Vilarasau de Ecozcue, 1967), also a functional homologization of at least the achromatic regions. It is attractive to trace the morphological evolution of the marked chromosome types (Figure 1) by postulating a few simple translocations. Since nothing is known on the information on the other parts of these chromosomes, one should approach the problem very carefully.

The frequency of associations in human metaphase plates is dependent on many technical factors, viz. the duration of the lymphocyte culture (Nankin, 1970) and the pre-treatment of the slides (Back \& Zang, 1969). This evidence should be taken into considcration in futurc research on primatc chromosome associations.

The present note is the first report on a broader project, supported financially by Grant No. 82-34 of the Netherlands Organization for the Advancement of Pure Research (Z.W.O.).

I am greatly obliged to Dr E. F. Jacobi (Artis Zoological Gardens, Amsterdam) and to Dr B. C. Kruyt (National Institute for Public Health, Bilthoven), who placed at our disposal she monkeys used in this study.

Thanks are due to Dr J. O. Van Hemel, for discussions on the problem and to Dr J. M. Van Brink and Dr B. Kiauta (all of this Institute) for reading the manuscript.

Mr D. Smit (Utrecht) made the illustrations in this paper.

Addendum. While this paper was in press, additional data were obtained on chromosome association in some prosimians and platyrrhines.

In metaphase plates of Galago crassicaudatus $(2 n=62)$, two medium sized acrocentric chromosomes were found to associate very frequently. Since the karyotype of this animal contains a large number of acrocentrics, it is not sure if the two chromosomes in question are homologues. No further elements seem to associate regularly.

A similar situation is met with in Galago senegalensis, though its karyotype $(2 n=38)$ differs essentially from that of the closely related $G$. crassicaudatus. The associating elements are approximately equal in length in both species. Since they are the only medium sized acrocentrics in the karyotype of $G$. senegalensis, they certainly are homologues.

In the third prosimian investigated, Perodicticus potto $(2 n=62$, karyotype very similar to that of Galago crassicaudatus), more complex associations were found. They consist of three or four acrocentrics of at least two pairs.

Finally, there is some evidence that the marked chromosomes of platyrrhines are involved in associations (based on material of Aotes trivirgatus, Saimiri sciureus and Leontocebus rosalia). Unlike the situation in catarrhines, where the marked chromosomes seem to be the only associating elements, in platyrrhines other associations of acrocentrics were found together with those of the marked chromosomes.

\section{References}

Back, E. \& Zang, K. D. (1969). Quantitative studies on the arrangement of human metaphase chromosomes. II. Influence of the preparation technique on the association pattern of the acrocentric chromosomes. Cytogenetics 8, 304-314.

de Boer L. E. M. (1971). Gytology of the Cercopithecidae with special reference to the karyotype of Cercopithecus hamlyni (Pocock, 1907). Proceedings of the Third International Congress of Primatology (in press).

Chiarelli, B. (1963). Primi resultati di recerche di genetica e cariolngia comparata in primati e loro interesse evolutivo. Rivista di Antropologica 50, 87-124.

Chiarelli, B. (1966a). Caryology and taxonomy of the Catarrhine monkeys. American Journal of Physical Anthropology 24, 155-170.

Chiarelli, B. (1966b). Marked chromosome in Catarrhine monkeys. Folia primatologica 4, 74-80. 
Egozcue, J. \& Vilarasau de Egozcue, M. (1967). The marked chromosomes of primates: Origin and cvolutionary significance. In (D. Starck, R. Schncidcr \& H. J. Kuhn, Eds). Neue Ergebnisse der Primatologie Stuttgart: G. Fischer.

Fernandez-Donoso, R., Lindsten, J. \& Norrby, E. (1970). The chromosomes of the Cynomologus macacque (Macaca fascicularis). Hereditas 65, 269-276.

Hamerton, J. L., Klinger, H. P., Mutton, D. E. \& Lang, E. M. (1963). 'The somatic chromosomes of the Hominoidea. Cytogenetics 2, 240-263.

van Hemel, J. O. (1971). Studies on human chromosome associations. Thesis. University of Utrccht.

Klinger, H. P. (1963). The somatic chromosomes of some primates (Tupaia glis, Tarsius bancanus, Cercocchus aterrimus, Symphalangus syndactylus). Cytogenetics 2, 140-151.

Nankin, H. R. (1970). In vitro alteration of satellite association and nucleolar persistence in mitotic human lymphocytes. Cytogenetics $9,42-51$.

Ohno, S., Trujillo, J. M., Kaplan, W. D. \& Kinosita, R. (1961). Nucleolus organizers in the causation of chromosomal anomalies in man. Lancet $65,123-126$.

Zang, K. D. \& Back, F. (1968). Quantitative studies on the arrangement of human metaphase chromosomes. I. Individual features in the association pattern of the acrocentric chromosomes of normal males and females. Cytogenetics 71, 455-470. 